\title{
Restoring Poissonian Images by a Combined First-Order and Second-Order Variation Approach
}

\author{
Le Jiang, ${ }^{1,2}$ Jin Huang, ${ }^{1}$ Xiao-Guang Lv, ${ }^{2}$ and Jun Liu ${ }^{1}$ \\ ${ }^{1}$ School of Mathematical Sciences, University of Electronic Science and Technology of China, Chengdu, Sichuan 610054, China \\ ${ }^{2}$ School of Science, Huaihai Institute of Technology, Lianyungang, Jiangsu 222005, China \\ Correspondence should be addressed to Xiao-Guang Lv; xiaoguanglv@126.com
}

Received 6 December 2012; Accepted 27 January 2013

Academic Editor: Kaleem R. Kazmi

Copyright (c) 2013 Le Jiang et al. This is an open access article distributed under the Creative Commons Attribution License, which permits unrestricted use, distribution, and reproduction in any medium, provided the original work is properly cited.

\begin{abstract}
The restoration of blurred images corrupted by Poisson noise is an important topic in imaging science. The problem has recently received considerable attention in recent years. In this paper, we propose a combined first-order and second-order variation model to restore blurred images corrupted by Poisson noise. Our model can substantially reduce the staircase effect, while preserving edges in the restored images, since it combines advantages of the first-order and second-order total variation. We study the issues of existence and uniqueness of a minimizer for this variational model. Moreover, we employ a gradient descent method to solve the associated Euler-Lagrange equation. Numerical results demonstrate the validity and efficiency of the proposed method for Poisson noise removal problem.
\end{abstract}

\section{Introduction}

Image restoration problem has been widely studied in the areas of image processing. The goal of image restoration is to reconstruct an approximation of an original image from a blurred and noisy one [1-4]. Over the last decades, most of the literature deal with the additive noise and multiplicative noise model [5-11]. Given a blurred and noisy image $g=$ $K u+v$ or $g=K u v$, where $u$ is the original image, $v$ represents the noise, and the blurring operator $K$ is a point spread function (PSF); the restoration problem involving the additive noise or multiplicative noise is to recover $u$ from the observed image $g$. The additive noise and multiplicative noise models have been extensively studied. We refer the reader to [12-19] and references therein for a review of various methods. In fact, there are other types of noise such as Poisson noise. In this paper, we consider the problem of seeking the approximations of original images from blurred images corrupted by Poisson noise. The restoration of blurred images corrupted by Poisson noise is an important task in various applications such as astronomical imaging, electronic microscopy, single-particle emission-computed tomography (SPECT), and positron emission tomography (PET) [20-24].
In a Poisson noise model, the recorded image $g$ is a realization of a random variable $\hat{g}$. A statistical model for $\hat{g}$ is given by

$$
\widehat{g} \sim \operatorname{Poiss}(K u+b),
$$

where $b>0$ is the expected value of the background which is assumed to be constant. The given data of the problem are the imaging operator $K$, the expected value of the background $b$, and the detected image $g$. It is known that the major difficulty for finding the original image $u$ of (1) is the models and algorithms developed for deconvolution of additive noise, or multiplicative noise images cannot be directly applied to the restoration of Poissonian images. Therefore, it is important to devise efficient and reliable algorithms for restoring blurred images corrupted by Poisson noise. The problem of restoration of Poissonian images has received considerable attention in recent years.

We note that, in a discrete setting, the intensity $g_{i}$ in the pixel $i$ is a random variable that follows a Poisson law of mean $(K u+b)_{i}$ where the structured matrix $K$ related to the boundary conditions is called a blurring matrix. Considering 
that these random variables are independent, the probability distribution of $g$, for given $K, u$, and $b$, can be written as

$$
\mathscr{L}(u)=P(g \mid u)=\prod_{i} \frac{\left[(K u+b)_{i}\right]^{g_{i}}}{g_{i} !} \exp ^{-(K u+b)_{i}}
$$

The use of Stirlings formula leads to the well-known Csiszár [25] $I$-divergence measure between $K x+b$ and $g$. Indeed, by taking the negative logarithm of the likelihood function, we obtain

$$
\mathscr{T}_{0}(u)=-\log [L(u)]=\sum_{i}(K u+b)_{i}-g_{i}+g_{i} \log \frac{g_{i}}{(K u+b)_{i}} .
$$

This measure generalizes the Kullback-Leibler divergence or cross-entropy measure to accommodate functions whose integrals are not constant, as they would be if they were probability distributions. Dropping the terms independent of $u$ in $\mathscr{L}(u)$, we obtain

$$
\mathscr{J}_{0}(u)=\sum_{i}(K u+b)_{i}-g_{i} \log (K u+b)_{i} .
$$

Therefore, the maximum likelihood estimator of the original image is the minimizer with respect to $u$ of the negative-log Poisson likelihood functional $\mathscr{F}_{0}(u)$.

As is well known, the structured matrix $K$ has many singular values of different orders of magnitude close to the origin. This makes the minimizer of (4) very sensitive to the noise in the right-hand side $g$. Thus a regularization method may be employed to compute the approximate solution that is less sensitive to noise than the naive solution. In general, regularization methods formulate the image restoration problem as a minimization problem of the form:

$$
\mathscr{J}(u)=\mathscr{J}_{0}(u)+\alpha \mathscr{F}_{R}(u),
$$

where $\mathscr{J}_{R}(u)$ is a regularization function and $\alpha>0$ is the regularization parameter that controls the balance between $\mathscr{J}_{0}$ and $\mathscr{J}_{R}$. We note that the regularization parameter $\alpha$ is an important quantity which controls the properties of the regularized solution, and $\alpha$ should therefore be chosen with care. Throughout the years a variety of parameter choice strategies such as the discrepancy principle, the L-curve, and generalized cross-validation (GCV) have been proposed for Poisson noise removal problem [26-28].

It is shown in [29] that the choice of the regularization function $\mathscr{J}_{R}(u)$ is related to the features of the images to be restored. The use of different regularization functions is an active research area. Probably one of the most popular regularization functions is the Tikhonov regularization function $\mathscr{F}_{R}(u)=(1 / 2)\|L u\|_{2}^{2}$, where $L$ is an auxiliary operator chosen among the identity and low-order differential operators; see [30] for more details. In [31], Landi and Piccolomini proposed a quasi-Newton projection method for deblurring Poisson-corrupted images after formulating the image restoration problem as a nonnegatively constrained minimization problem where the objective function is the sum of the Kullback-Leibler divergence, used to express fidelity to the data in the presence of Poisson noise, and of a Tikhonov regularization term. Their numerical results show the potential of the method both in terms of relative error reduction and computational efficiency. In $[32,33]$, the authors developed a cost functional which incorporates the statistics of the noise in the image data and Tikhonov regularization to induce stability and employed an efficient hybrid gradient projection-reduced Newton (GPRNCG) method for the problem of restoration of Poissonian images. Finally, they gave a comparison between the Richardson-Lucy [34, 35] iterative regularization method and GPRNCG algorithm for the restoration of blurred images corrupted by Poisson noise. By the measures of performance used in this comparison, GPRNCG was more efficient than Richardson-Lucy iteration.

It is known that Tikhonov regularization estimate is similar to low-pass filtering; therefore, it produces a smoothing effect on the restored images; that is, it penalizes edges, which is not a good approximation of the original image if it contains edges. To overcome this shortcoming, Rudin et al. [5] proposed a total variation- (TV-) based regularization technique, which preserves the edge information in the restored image. In the case of TV regularization, the estimated solution is obtained by minimizing the object function:

$$
\mathscr{J}(u)=\mathscr{J}_{0}(u)+\alpha\|u\|_{\mathrm{TV}}
$$

where $\|u\|_{\mathrm{TV}}=\|\nabla u\|_{1}$ is the discrete TV regularization term. It is shown in [36] that the TV penalty function acts more like a model selection device by identifying a small number of critical points at which $u$ is allowed to jump. The number of these selected jump points is controlled by $\alpha$. Especially, if $u$ is piecewise constant with a finite number of jump discontinuities, then $\|u\|_{\mathrm{TV}}$ gives the sum of magnitudes of the jumps.

In [21], Bardsley and Luttman considered the TV regularization for poissonian images and showed that the problem of computing the minimizer of the resulting TV-penalized Poisson likelihood functional is well posed. They then proved that as the errors in the data and in the blurring operator tend to zero, the resulting minimizer converges to the minimizer of the exact likelihood function. Finally, the practical effectiveness of the approach is demonstrated on synthetically generated data, and a nonnegatively constrained, projected quasi-Newton method was introduced. In [22], Figueiredo and Bioucas-Dias proposed an approach to deconvolving Poissonian images with the TV function, which is based upon an alternating direction optimization method. Using standard convex analysis tools, they presented sufficient conditions for existence and uniqueness of solutions of these optimization problems. In [23], Setzer et al. considered the restoration of blurred images corrupted by Poisson noise by minimizing an energy functional consisting of the KullbackLeibler divergence as similarity term and the TV regularization term. Their minimizing algorithm uses alternating split Bregman techniques which can be reinterpreted as Douglas-Rachford splitting applied to the dual problem. In contrast to recently developed iterative algorithms, their algorithm contains no inner iterations and produces nonnegative images. The high efficiency of their algorithm in comparison 
to other recently developed algorithms is demonstrated by artificial and real-world numerical examples. We refer the reader to $[24,37]$ and references therein for other efficient methods solving the restoration of blurred images corrupted by Poisson noise with the TV function.

Although the total variation regularization is extremely popular in a variety of applications, it also gives rise to some undesired effects. Both from a theoretical and experimental point of view, it has been shown that the TV norm transforms smooth signal into piecewise constants, the socalled staircase effect. Staircase solutions fail to satisfy the evaluation of visual quality, and they can develop false edges that do not exist in the true image. To attenuate the staircase effect, there is a growing interest in the literature for replacing the TV norm by a high-order TV norm. The motivation behind such attempt is to restore potentially a wider range of images, which comprise more than merely piecewise constant regions. The majority of the high-order norms involve second-order differential operators because piecewise-vanishing second-order derivatives lead to piecewise-linear solutions that better fit smooth intensity changes; see [38] for more details.

Second-order regularization schemes have been considered so far in the literature mainly for dealing with the staircase effect while preserving the edge information in the restored image. There are two classes of second-order regularization methods for image restoration problems. The first class combines a second-order regularizer with the TV norm. For example, a technique in $[39,40]$ combining the TV filter with a fourth-order PDE filter was proposed to preserve edges and at the same time avoid the staircase effect in smooth regions for noise removal. In [41], Papafitsoros and Schönlieb considered a high-order model involving convex functions of the TV and the TV of the first derivatives for image restoration problems and used the split Bregman method to solve numerically the corresponding discretized problem. Chan et al. proposed a second-order model to substantially reduce the staircase effect, while preserving sharp jump discontinuities for image restoration in [42]. The second class employs a second-order regularizer in a standalone way. The secondorder regularizer was first proposed in [43] for additive noise removal in medical magnetic resonance images. The LLT model presented in [43] can overcome the staircase effect that occurs with the TV norm filter and better preserve the fine details in nonblocky images. In order to test its practical potential the authors have applied their method to a wide range of real images, including structural and functional MRI data sets. The main strength of their method was the ability to process signals with a smooth change in the intensity value. Numerical results have shown that, compared with some related partial differential equation (PDE) models, the LLT model is rather robust in removing noise and handling edges. In $[44,45]$, the authors reconsidered the fourth-order PDE model for additive noise removal and employed the dual algorithm of Chambolle for solving the high-order problems.

In order to get restored images with edge preserved and simultaneously smooth region reconstructed for Poisson noise removal, it is natural to utilize a combined first-order and second-order total variation technique. In this paper, we consider to modify the total variation model by adding a high-order functional for restoring blurred images corrupted by Poisson noise. Our model can substantially reduce the staircase effect, while preserving edges in the restored images, since it combines advantages of the first-order and second-order total variation. We study the issues of existence and uniqueness of a minimizer for this variational model. Moreover, we employ a gradient descent method to solve the associated Euler-Lagrange equation. Some numerical results demonstrate the validity and efficiency of the proposed method for Poisson noise removal problem.

The organization of this paper is outlined as follows. In the next section, we present a combined first-order and second-order variation model to restore blurred images corrupted by Poisson noise and study the issues of existence and uniqueness of a minimizer for this variational model. A gradient descent method to solve the associated EulerLagrange equation is presented in Section 3. Some numerical experiments are given to illustrate the performance of the proposed algorithm in Section 4. We give concluding remarks in the last section.

\section{A Combined First-Order and Second-Order Variation Model}

In the Bayesian approach, a prior probability density $P_{u}(u)$ for $u$ is also specified, and the posterior density:

$$
P_{u}(u \mid g)=\frac{P_{g}(g \mid u) P_{u}(u)}{P_{g}(g)},
$$

given by Bayes's law, is maximized with respect to $u$. In (7), $P_{g}(g)$ defines the prior probability distribution of the measurements $g$, which are given by measurements, and the likelihood function $P_{g}(g \mid u)$ is the conditional probability of observing a fixed $g$ for a variable $u$. The maximizer of $P_{u}(u \mid g)$ is called the maximum a posteriori (MAP) estimate. Since $P_{g}(g)$ does not depend on $u$, it is not needed in the computation of the MAP estimate and thus can be ignored. Taking the natural logarithm of (7) and dropping the terms independent of $u$, we obtain that maximizing (7) is equivalent to minimizing

$$
E(u)=\mathscr{J}_{0}(u)-\log P_{u}(u)
$$

with respect to $u$, where $\mathscr{F}_{0}(u)=\int_{\Omega}((K u+b)-g \log (K u+$ b)) $d x d y$. It is clear that $-\log P_{u}(u)$ corresponds to the regularization term in the classical penalized likelihood approach to regularization. However in the Bayesian setting, $P_{u}(u)$ is the probability density known as the prior from which the unknown $u$ is assumed to arise. Thus the prior knowledge regarding the characteristics of $u$ can be formulated in the form of a probability density $P_{u}(u)$, and this yields to a natural, and statistically rigorous, motivation for the regularization method [27].

Standard Tikhonov regularization corresponds to the following choice of the prior:

$$
P_{u}(u)=\exp ^{-(\alpha / 2)\|u\|_{2}^{2}}
$$


This corresponds to the assumption that the prior for $u$ is a zero-mean Gaussian random variable with covariance matrix $\alpha^{-1} I$, which has the effect of penalizing reconstructions with large $L_{2}$ norm. For $L_{2}$ norm of the gradient regularization, the penalty has the similar form

$$
P_{u}(u)=\exp ^{-(\alpha / 2)\|\nabla u\|_{2}^{2}}
$$

where $\nabla$ is the gradient operator. The use of this regularization function has the effect of penalizing reconstructions that are not smooth. As an alternative to Tikhonov regularization including standard Tikhonov regularization and gradient regularization for Poisson noise removal problem, total variation regularization is another regularization technique that allows for the presence of sharp edges in the resulting reconstruction. In the case of total variation regularization, we have

$$
P_{u}(u)=\exp ^{-\alpha\|\nabla u\|_{1}},
$$

where $\|\nabla u\|_{1}=\int_{\Omega} \sqrt{u_{x}^{2}+u_{y}^{2}} d x d y$.

Optimization techniques such as alternation direction method of multipliers (ADMM) and alternating split Bregman algorithm were proposed for the restoration of blurred images corrupted by Poisson noise by minimizing an energy functional consisting of the Kullback-Leibler divergence as the similarity term and the TV regularization term; see [2124] for more details. In the other hand, some Newton-related gradient methods were proposed for deblurring Poissoncorrupted images by formulating the image restoration problem as a nonnegatively constrained minimization problem where the objective function is the sum of the KullbackLeibler divergence, used to express fidelity to the data in the presence of Poisson noise, and of a Tikhonov regularization term; see [31-33] for more details.

To attenuate the staircase effect in the TV regularization, we consider a combined first-order and second-order variation model to restore blurred images corrupted by Poisson noise in this work. In the proposed model, the prior probability density is of the form:

$$
P_{u}(u)=\exp ^{-\alpha\left(\theta\|\nabla u\|_{1}+(1-\theta)\left\|\nabla^{2} u\right\|_{1}\right)},
$$

where $\left\|\nabla^{2} u\right\|_{1}=\int_{\Omega} \sqrt{u_{x x}^{2}+u_{x y}^{2}+u_{y x}^{2}+u_{y y}^{2}} d x d y$. It leads to present the following functional for restoring blurred images corrupted with Poisson noise:

$$
\begin{aligned}
\min _{u}\{E(u)= & \mathscr{J}_{0}(u)+\alpha \mathscr{J}_{R}(u) \\
=\int_{\Omega}( & (K u+b)-g \log (K u+b) \\
& \left.\left.+\alpha\left(\theta|\nabla u|+(1-\theta)\left|\nabla^{2} u\right|\right)\right) d x d y\right\},
\end{aligned}
$$

where the parameter $\theta \in[0,1]$ is used to control the balance between the edges and the smooth surface and $\alpha$ is the regularization parameter which measures the tradeoff between the fidelity term and the regularized term. The parameter $\theta$ can be computed by using the information of the edges and smooth regions of the resulting image obtained by smoothing the observed image $g$ with the low-pass filters such as the median filter and the Gauss filter.

The functional $E(u)$ in (13) is defined on the set of $u \in$ $B V(\Omega) \cap B V^{2}(\Omega)$ such that $\log u \in L_{1}(\Omega)$; in particular, $u$ must be positive almost everywhere. Some basic notations and properties concerning the $B V$ space and $B V^{2}$ space can be found in $[39,40]$. Motivated by [46], we have the following result to show the existence and uniqueness of the minimizer for the model (13).

Theorem 1. Let $\Omega$ be a bounded, open subset of $\mathbb{R}^{2}$ with Lipschitz boundary. Assume that $g$ is a positive bounded function and $K$ is positive definite. Then $E(u)$ for $u \in B V(\Omega) \cap$ $B V^{2}(\Omega)$ such that $\log u \in L_{1}(\Omega)$ has a unique minimizer for the model (13).

Proof. First, a straightforward computation can show that $\mathscr{F}_{0}(u)$ is bounded below by $g-g \log g$. Since the penalty function $\mathscr{J}_{R}(u) \geq 0$, we obtain that $E(u)$ is bounded below. Thus, we can choose a sequence $\left\{u^{n}\right\}$ such that $E\left(u^{n}\right) \geq$ $E\left(u^{n+1}\right)$. Hence, $\mathscr{J}_{0}\left(u^{n}\right)$ and $\mathscr{J}_{R}\left(u^{n}\right)$ are bounded. Using Jensen's inequality, we have

$$
\mathscr{J}_{0}\left(u^{n}\right) \geq\left\|u^{n}\right\|_{1}-\|g\| \log \left\|u^{n}\right\|_{1},
$$

which indicates that $\left\|u^{n}\right\|_{1}$ is bounded. This and the boundedness of $\mathscr{J}_{R}\left(u^{n}\right)$ yields that the sequence $\left\{u^{n}\right\}$ is bounded in $B V(\Omega) \cap B V^{2}(\Omega)$. Following the compactness of $L_{1}(\Omega)$ in $B V(\Omega) \cap B V^{2}(\Omega)$, we deduce that there exists $u^{*} \epsilon$ $B V(\Omega) \cap B V^{2}(\Omega)$ such that a subsequence $\left\{u^{n_{k}}\right\}$ converges to $u^{*}$ pointwise almost everywhere in $L_{1}$. By the lower semicontinuity of the $B V(\Omega) \cap B V^{2}(\Omega)$ space, we obtain that $\lim \inf \mathscr{J}_{R}\left(u^{n_{k}}\right) \geq \mathscr{J}_{R}\left(u^{*}\right)$. Since $\mathscr{J}_{0}\left(u_{n_{k}}\right)$ is bounded below, we get that $\lim \mathscr{J}_{0}\left(u^{n_{k}}\right) \geq \mathscr{J}_{0}\left(u^{*}\right)$ by using Fatou's lemma. Therefore, $\lim \inf E\left(u^{n_{k}}\right) \geq E\left(u^{*}\right)$ and $u^{*}$ minimizes $E(u)$ for all $u \in B V(\Omega) \cap B V^{2}(\Omega)$ such that $\log u \in L_{1}(\Omega)$.

Since $K$ is positive definite and $g$ is positive, it immediately follows that $\mathscr{J}_{0}(u)$ is strictly convex. Obviously, $\mathscr{J}_{R}(u)$ is a convex function. We conclude that $E(u)$ is strictly convex, as the sum of a convex function and of a strictly convex function. Therefore, the minimizer $u^{*}$ is unique.

\section{Computational Method}

In this section, our aim is to propose a time-marching gradient descent method for computing the minimizer of the model (13). From [5, 39, 40] we know that minimizing (13) 
for a given constant $\theta$ yields the associate Euler-Lagrange equation:

$$
\begin{aligned}
0= & -\theta \nabla \cdot\left(\frac{\nabla u}{|\nabla u|}\right) \\
& +(1-\theta)\left[\left(\nabla \cdot\left(\frac{\nabla u_{x}}{\left|\nabla^{2} u\right|}\right)\right)_{x}+\left(\nabla \cdot\left(\frac{\nabla u_{y}}{\left|\nabla^{2} u\right|}\right)\right)_{y}\right] \\
& +K^{T} \frac{K u+b-g}{\alpha(K u+b)}
\end{aligned}
$$

with the boundary conditions

$$
\begin{gathered}
\nabla u \cdot N=0, \quad \nabla u_{x} \cdot N=0, \quad \nabla u_{y} \cdot N=0, \\
\nabla \cdot\left(\frac{\nabla u_{x}}{\left|\nabla^{2} u\right|}\right) n_{1}=0, \quad \nabla \cdot\left(\frac{\nabla u_{y}}{\left|\nabla^{2} u\right|}\right) n_{2}=0,
\end{gathered}
$$

where $N=\left(n_{1}, n_{2}\right)$ denotes the unit outernormal vector of $\partial \Omega$.

Using the gradient descent method, we are able to derive the associated heat flow for the model (13):

$$
\begin{aligned}
\frac{\partial u}{\partial t}=\theta & \cdot\left(\frac{\nabla u}{|\nabla u|}\right)-(1-\theta) \\
\times & {\left[\left(\frac{u_{x x}}{\left|\nabla^{2} u\right|}\right)_{x x}+\left(\frac{u_{x y}}{\left|\nabla^{2} u\right|}\right)_{y x}\right.} \\
& \left.+\left(\frac{u_{y x}}{\left|\nabla^{2} u\right|}\right)_{x y}+\left(\frac{u_{y y}}{\left|\nabla^{2} u\right|}\right)_{y y}\right]-K^{T} \frac{K u+b-g}{\alpha(K u+b)} .
\end{aligned}
$$

In this work, we use the finite difference scheme to discretize (17); see $[5,39,40]$ for more details. Denoting the step space by $h=1$, we employ the following discretization used in the implementations; see Table 1.

In the discretization, the notation $m[a, b]=((\operatorname{sgn} a+$ $\operatorname{sgn} b) / 2) \cdot \min (|a|,|b|)$ and $\delta>0$ is near 0 . Denoting the time step by $\tau$, we get the following explicit computational scheme:

$$
\begin{aligned}
u^{k+1}= & u^{k}+\tau \theta\left[D_{x}^{-} \frac{D_{x}^{+} u^{k}}{\left|D_{x} u^{k}\right|}+D_{y}^{-} \frac{D_{y}^{+} u^{k}}{\left|D_{y} u^{k}\right|}\right]+\tau(1-\theta) \\
\times & {\left[D_{x x}\left(\frac{D_{x x} u^{k}}{\left|D^{2} u^{k}\right|}\right)+D_{y x}^{-}\left(\frac{D_{x y}^{+} u^{k}}{\left|D^{2} u^{k}\right|}\right)\right.} \\
& \left.+D_{x y}^{+}\left(\frac{D_{y x}^{-} u^{k}}{\left|D^{2} u^{k}\right|}\right)+D_{y y}\left(\frac{D_{y y} u^{k}}{\left|D^{2} u^{k}\right|}\right)\right] \\
& -\tau K^{T} \frac{K u^{k}+b-g}{\alpha\left(K u^{k}+b\right)} .
\end{aligned}
$$

Now we discuss the choice of the weighting parameter $\theta$ in (18). Due to the strengths and weaknesses of the firstorder and second-order variation approach, it is desirable
TABLE 1

\begin{tabular}{lc}
\hline$D_{x}^{ \pm} u_{i, j}$ & $\pm\left(u_{i \pm 1, j}-u_{i, j}\right)$ \\
$D_{y}^{ \pm} u_{i, j}$ & $\pm\left(u_{i, j \pm 1}-u_{i, j}\right)$ \\
$D_{x x} u_{i, j}$ & $D_{x}^{ \pm} u_{i, j}-D_{x}^{ \pm} u_{i-1, j}$ \\
$D_{x y}^{ \pm} u_{i, j}$ & $\pm\left[D_{x}^{ \pm} u_{i, j}-D_{x}^{ \pm} u_{i-1, j}\right]$ \\
$D_{y x}^{ \pm} u_{i, j}$ & $\pm\left[D_{y}^{ \pm} u_{i \pm 1, j}-D_{y}^{ \pm}\left(u_{i, j}\right)\right]$ \\
$D_{y y} u_{i, j}$ & $D_{x}^{ \pm} u_{i, j}-D_{x}^{ \pm} u_{i, j-1}$ \\
$\left|D_{x} u_{i, j}\right|$ & $\sqrt{\left(D_{x}^{+} u_{i, j}\right)^{2}+\left(m\left[D_{y}^{+} u_{i, j}, D_{y}^{-} u_{i, j}\right]\right)^{2}+\delta}$ \\
$\left|D_{y} u_{i, j}\right|$ & $\sqrt{\left(m\left[D_{x}^{+} u_{i, j}, D_{x}^{-} u_{i, j}\right]\right)^{2}+\left(D_{y}^{+} u_{i, j}\right)^{2}+\delta}$ \\
$\left|D^{2} u_{i, j}\right|$ & $\sqrt{\left(D_{x x} u_{i, j}\right)^{2}+\left(D_{x y}^{+} u_{i, j}\right)^{2}+\left(D_{y x}^{+} u_{i, j}\right)^{2}+\left(D_{y y} u_{i, j}\right)^{2}+\delta}$ \\
\hline
\end{tabular}

that the weighting parameter $\theta=1$ along edges and in flat regions, emphasizing the restoration properties for the firstorder total variation. To emphasize the restoration properties of the second-order total variation in smooth regions, we want $0 \leq \theta<1$. Specially, the resulting algorithm of (18) is just the TV regularization method for Poisson noise removal problem when $\theta=1$, while the resulting algorithm is the high-order TV regularization method when $\theta=0$. Usually, we may compute the parameter $\theta$ by using the information of the edges and smooth regions of the resulting image obtained by smoothing the observed image $g$ with the low-pass filters such as the median filter and the Gauss filter. We have carried out some numerical experiments. We observe that the fixed $\theta$ obtained by computing the observed image can give very good results. In this work, in order to detect edges and smoothing regions much better, we adopt the method for updating $\theta$ as proposed in [40]. The results obtained by carrying out various numerical examples show that the updating procedure behaves better for our model than the fixed $\theta$. It is because, as the iteration proceeds, the edges and smoothing regions of recovered image are closer to the original image, then the parameter $\theta$ computed by the updating scheme can be better suitable for restoration. So we employ the method in [40] for updating $\theta$ in our numerical experiments. More precisely, suppose that $u^{k}$ is the $k$ th iterative solution, and we update the parameter $\theta$ as follows:

$$
\begin{aligned}
& \theta_{i, j} \\
& = \begin{cases}1, & \text { if } \frac{\left|\nabla u_{i, j}^{k}\right|}{\max _{i, j}\left(\left|\nabla u_{i, j}^{k}\right|\right)} \\
\frac{1}{2} \cos \left(\frac{2 \pi\left|\nabla u_{i, j}^{k}\right|}{\operatorname{cmax}_{i, j}\left(\left|\nabla u_{i, j}^{k}\right|\right)}\right)+\frac{1}{2}, & \text { otherwise, }\end{cases}
\end{aligned}
$$

where $0 \ll c<1$, and it means that only the absolute largest jumps are unaffected of the high-order regularization. As reported in [40], for large and small values of $\left|\nabla u_{i, j}^{k}\right|$ the parameter $\theta$ is closer to 1 , and for intermediate values of $\left|\nabla u_{i, j}^{k}\right|$ the parameter $\theta$ approaches 0 , which means that 
(1) Input $g, K, b$, MaxIter, $\alpha$ and $\tau$. Initialize $u^{0}$

(2) For $k=1$ : MaxIter

compute $u^{k}$ using the explicit scheme (18).

update the parameter $\theta$ according to (19). end for

Algorithm 1: The time-marching gradient descent algorithm for solving (13).

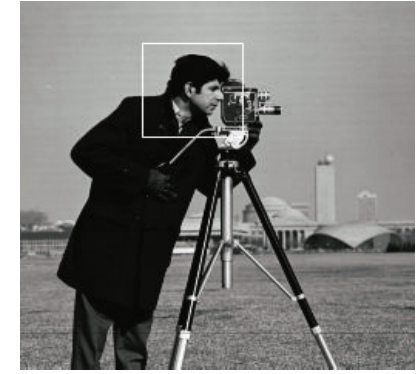

(a) True image

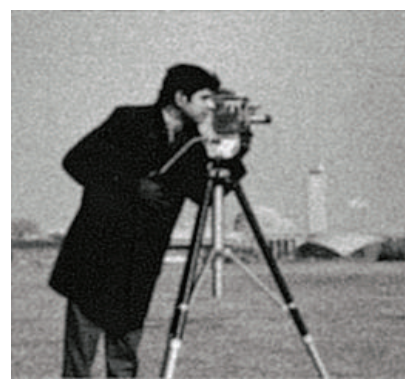

(c) Tikhonov

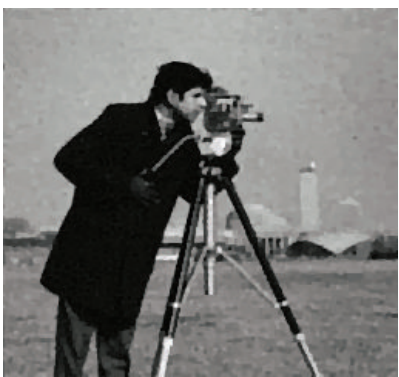

(d) $\mathrm{TV}$

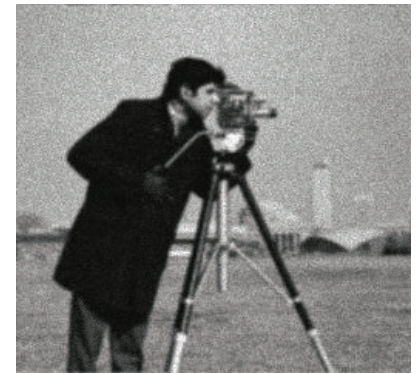

(b) Degraded image

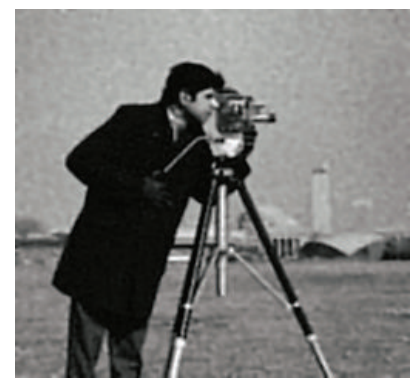

(e) HTV

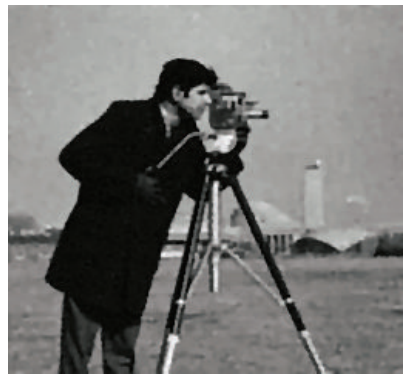

(f) Hybrid TV

FIgURE 1: The "Cameraman" image is degraded by the Gaussian blur and Poisson noise.

the high-order filter dominates the computation and the staircase effect is suppressed. Since only small jumps should be suppressed with the high-order regularization, it is a good choice for $c=1 / 8$.

We are now in a position to describe the time-marching gradient descent algorithm (Algorithm 1) for restoring blurred images corrupted by Poisson noise.

\section{Numerical Experiments}

In this section, we present some numerical results to illustrate the performance of the proposed model for Poisson noise removal problem. We compare our model with the one proposed in [21] (TV method), the one proposed in [31] (Tikhonov method), and the one proposed in [47] (HTV method). All computations of the present paper were carried out in Matlab 7.10. The results were obtained by running the Matlab codes on an Intel(R) Core(TM) i3 CPU $(2.27 \mathrm{GHz}$, $2.27 \mathrm{GHz}$ ) computer with RAM of $2048 \mathrm{M}$.

The quality of the restoration results with different methods is compared quantitatively by using the signal-tonoise ratio (SNR), the relative error (ReErr), and structural SIMilarity index (SSIM). They are defined as follows:

$$
\begin{gathered}
\mathrm{SNR}=20 \log _{10}\left(\frac{\left\|u_{\text {true }}\right\|_{2}}{\left\|u^{k}-u_{\text {true }}\right\|_{2}}\right), \\
\text { ReErr }=\frac{\left\|u^{k}-u_{\text {true }}\right\|_{2}}{\left\|u_{\text {true }}\right\|_{2}},
\end{gathered}
$$

where $u_{\text {true }}$ and $u^{k}$ are the ideal image and the restored image, respectively, and

$$
\operatorname{SSIM}=\frac{\left(2 \mu_{1} \mu_{2}+C_{1}\right)\left(2 \sigma_{12}+C_{2}\right)}{\left(\mu_{1}^{2}+\mu_{2}^{2}+C_{1}\right)\left(\sigma_{1}^{2}+\sigma_{2}^{2}+C_{2}\right)},
$$

where $\mu_{1}$ and $\mu_{2}$ are averages of $u_{\text {true }}$ and $u^{k}$, respectively, $\sigma_{f}$ and $\sigma_{\tilde{f}}$ are the variance of $u_{\text {true }}$ and $u^{k}$, respectively, and $\sigma_{12}$ is the covariance of $u_{\text {true }}$ and $u^{k}$. The positive constants $C_{1}$ and $C_{2}$ can be thought of as stabilizing constants for near-zero denominator values. The SSIM is a well-known quality metric used to measure the similarity between two images. The SSIM method was developed by Wang et al. [48] and is based on three specific statistical measures that are much closer to how the human eye perceives differences between images. In the following experiments, we will use SSIM map to reveal areas 


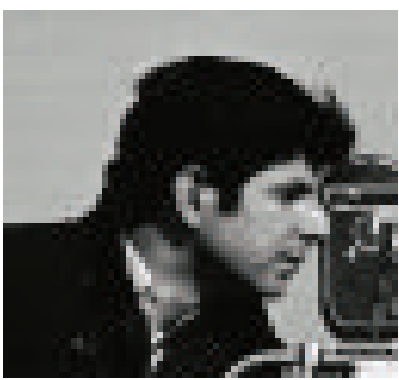

(a) True

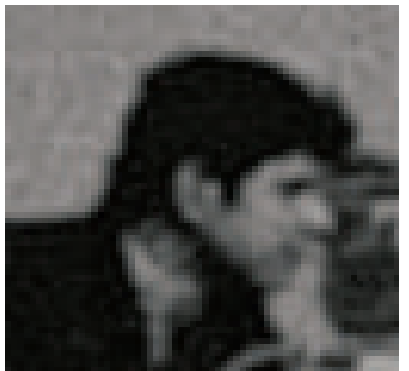

(c) Tikhonov

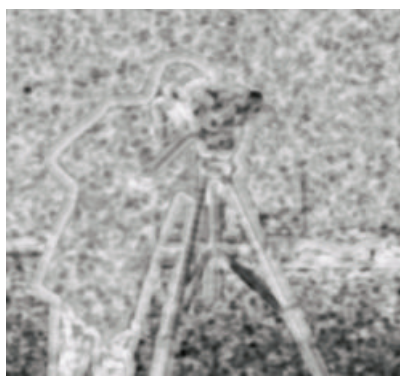

(g) Tikhonov

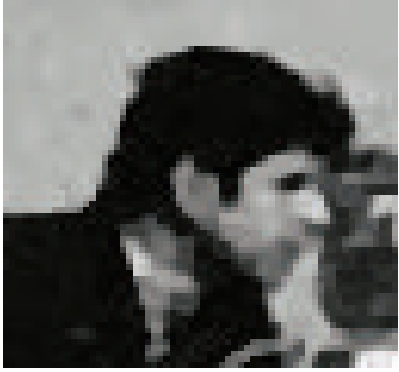

(d) $\mathrm{TV}$

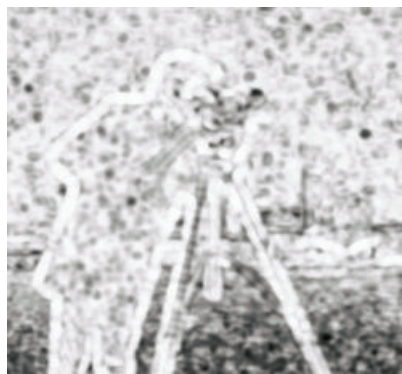

(h) $\mathrm{TV}$

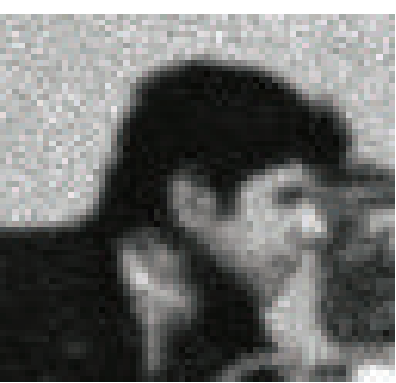

(b) Degraded

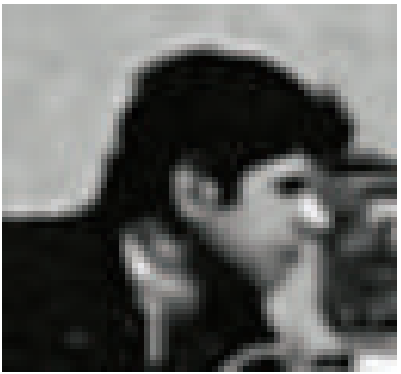

(e) HTV

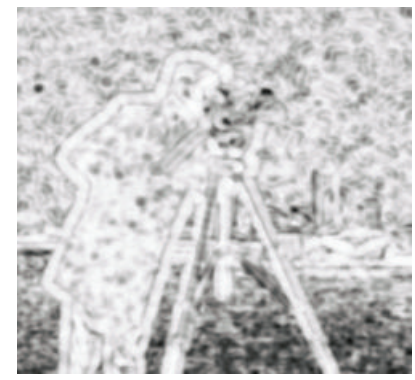

(i) HTV

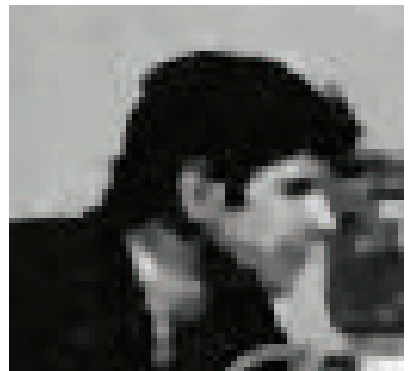

(f) Hybrid TV

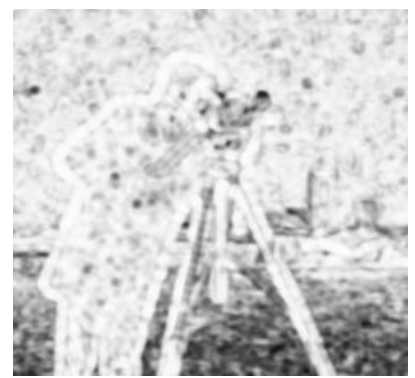

(j) Hybrid TV

FIGURE 2: Comparison of a small portion of the restored images and SSIM maps for Example 1.

of high or low similarity between two images, the whiter SSIM map, and the closer between the two images.

In order to give a good comparison, we employ the timemarching gradient descent algorithm for the four models. In the time-marching gradient descent algorithm, the parameter $\epsilon=10^{-4}$ is introduced to avoid divisions by zero. The initial guess is chosen to be the degraded image in all tests. We set the step size $\tau$ as 0.1 in order to obtain a stable iterative procedure. We terminate the iterations for the methods and accept $u^{k}$ as the computed approximation of the ideal image as soon as the maximum number of allowed outer iterations has been carried out or the relative differences between consecutive iterates $u^{1}, u^{2}, u^{3}, \ldots$ satisfy

$$
\frac{\left\|u^{k+1}-u^{k}\right\|_{2}}{\left\|u^{k+1}\right\|_{2}}<10^{-4} \text {. }
$$

In addition, we carry out many experiments with different regularization parameters in the models, andthe one with the best results is presented in this work. In this way, we have a fair comparison since we compare the restorations for four different methods.
In the first experiment, we consider the "Cameraman" image of size $256 \times 256$. The degraded image in Figure 1(b) is obtained by performing the blurring operation psfGauss $(5,1)$ proposed in [49] on the original image in Figure 1(a) with the background $b=10$ and adding the Poisson noise. Note that there is no parameter associated with the Poisson noise, but the noise magnitude depends on the absolute image intensities. The amount of noise in a region of the image increases with the intensity of the image there. The restored images by all four methods are shown in Figures 1(c)-1(f). From these figures, compared with the tikhonov regularization method, the TV regularization method, and the high TV regularization method, the proposed approach yields better results in image restoration since it avoids the staircase effect of the general TV methods, and the edges are preserved as well as the general TV methods. In Figures 2(c)-2(f), we have enlarged some details of the four restored images. As it is seen in the zoomed parts, the proposed method outperforms the other three methods. In Figures 2(c)-2(f), we also present the SSIM maps of the restored images in Figures $2(\mathrm{~g})-2(\mathrm{j})$. Note that the SSIM maps of the restored image by the proposed method are whiter than the 


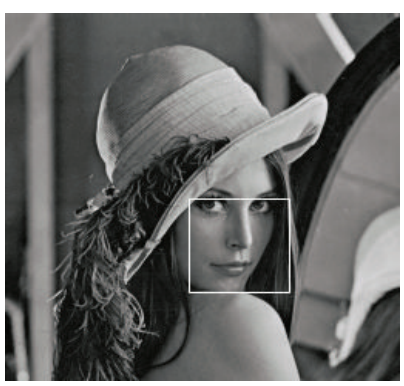

(a) True image

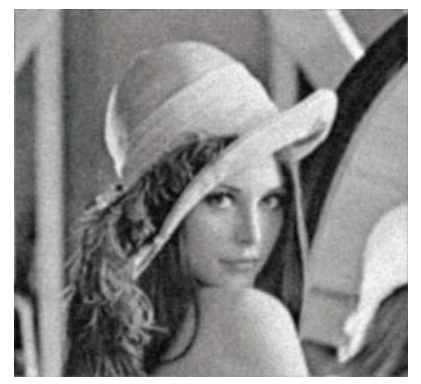

(c) Tikhonov

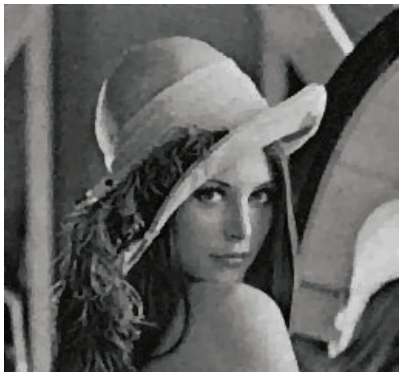

(d) TV

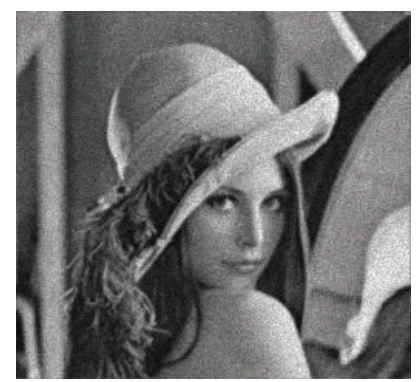

(b) Degraded image

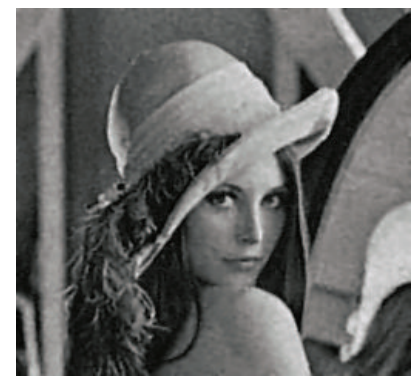

(e) HTV

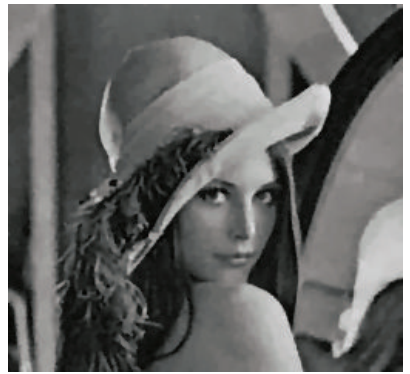

(f) Hybrid TV

Figure 3: The "Barbara" image is degraded by the Moffat blur and Poisson noise.

TABLE 2: Restoration results with different methods for Example 1, Example 2, and Example 3.

\begin{tabular}{|c|c|c|c|c|}
\hline & Method & Parameter & SNR & ReErr \\
\hline \multirow{4}{*}{ Example 1} & Tikhonov & $\alpha=400$ & 8.725 & 0.2657 \\
\hline & TV & $\alpha=65$ & 14.321 & 0.0895 \\
\hline & HTV & $\alpha=80$ & 14.001 & 0.0930 \\
\hline & Hybrid TV & $\alpha=60$ & 14.538 & 0.0871 \\
\hline \multirow{4}{*}{ Example 2} & Tikhonov & $\alpha=500$ & 9.728 & 0.2223 \\
\hline & TV & $\alpha=30$ & 15.811 & 0.0758 \\
\hline & HTV & $\alpha=80$ & 16.022 & 0.0740 \\
\hline & Hybrid TV & $\alpha=40$ & 16.323 & 0.0718 \\
\hline \multirow{4}{*}{ Example 3} & Tikhonov & $\alpha=400$ & 6.924 & 0.4369 \\
\hline & TV & $\alpha=50$ & 13.675 & 0.2007 \\
\hline & HTV & $\alpha=15$ & 11.566 & 0.2559 \\
\hline & Hybrid TV & $\alpha=70$ & 14.912 & 0.1690 \\
\hline
\end{tabular}

other methods; that is, our method can get better results. In Table 2, we compare their restoration results in SNRs and ReErrs. We observe from Table 2 that both the SNR and ReErr values of the restored images by the proposed method are better than those by the other three methods.

Moffat blur is considered in the second example. It is known that the PSF of an astronomical telescope is often modeled by the Moffat function. The $256 \times 256$ "Lena" image shown in Figure 3(a) is degraded by the Moffat blur psfMoffat $(5,2,5)$ proposed in $[50]$ with $b=10$ and the Poisson noise. The information of restored images by the four methods is displayed in Figures 3(c)-3(f). From the visual quality of restored images, the proposed regularization method is better than the other three methods. In Figures 4(c)-4(f), we display the zoom parts of the restored images (the part shown as the white rectangle in Figure 3(a)). From Figures 4(c)-4(f), we see that the restored image obtained by our method has more details than those by the other methods. The comparison of SSIM maps shown in Figures $4(\mathrm{~g})-4(\mathrm{j})$ also proves that our method can get better results. We report the SNR and RelErr values in Table 2 . From the table, we know that our method behaves much better.

In the third example, the $256 \times 256$ original astronomical object shown in Figure 5(a) is degraded by the given PSF in Figure 5(b) and the Poisson noise. The degraded image is displayed in Figure 5(c). The relative error between the noisy image and the original image is 0.3150 .

In Figures 5(e)-5(h), we show the restored images by the four different methods. From these figures, we observe that the restored image by our model is more better than the other models in terms of the staircase effect and edge preservation. In addition, we plot the choice of $\theta$ in Figure 5(d). From 


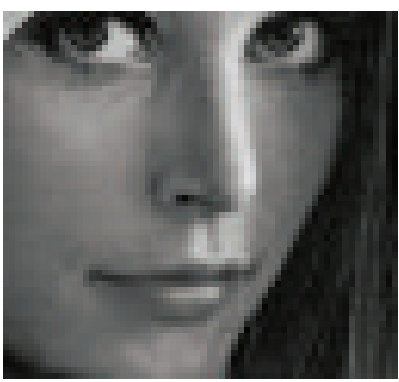

(a) True

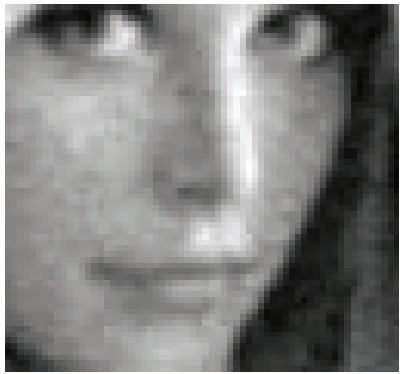

(c) Tikhonov

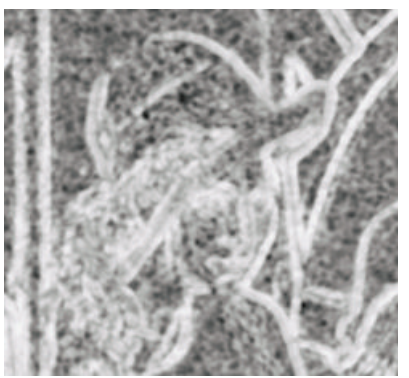

(g) Tikhonov

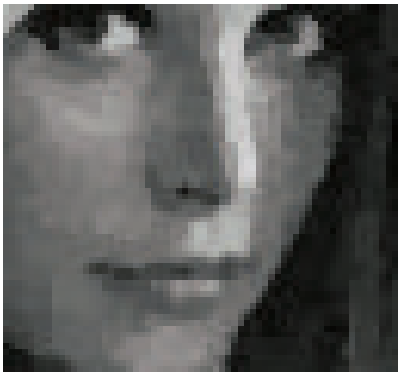

(d) $\mathrm{TV}$

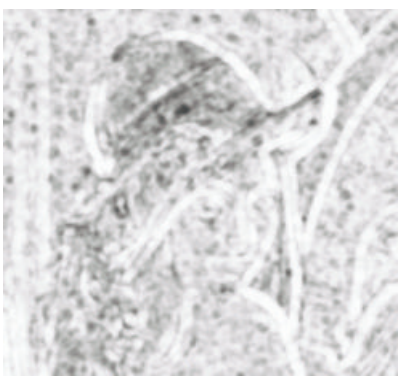

(h) TV

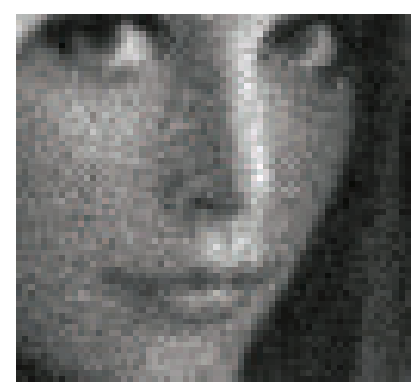

(b) Degraded

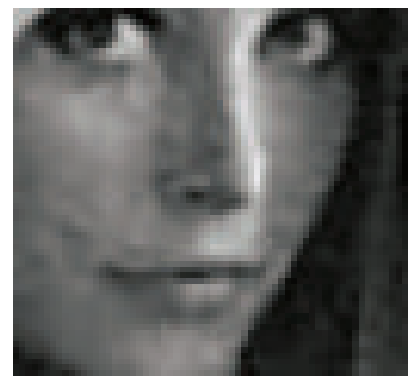

(e) HTV

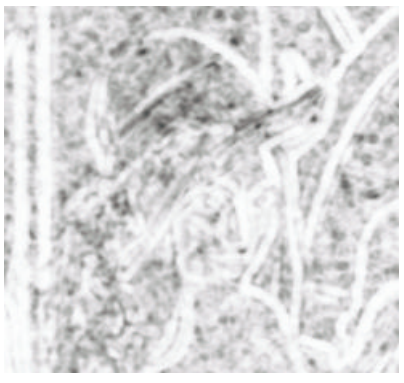

(i) HTV

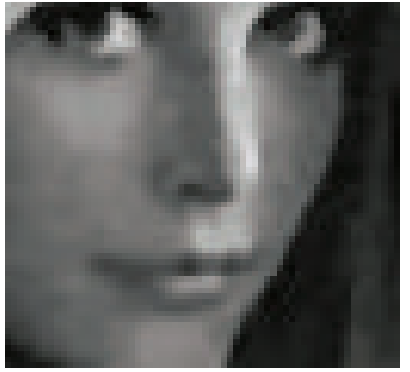

(f) Hybrid TV

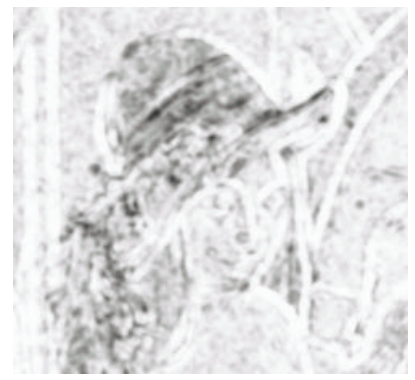

(j) Hybrid TV

FIgURE 4: Comparison of a small portion of the restored images and SSIM maps for Example 2.

the figure, we see that only small jumps are suppressed with the second-order regularization. The SNRs and relative errors are reported in Table 2. The SNR by our method is higher than those by the other methods, and the relative error by our method is smaller than those by the other methods. From these figures and Table 2, we observe that the proposed method outperforms the tikhonov regularization method, the TV regularization method, and the high TV regularization method.

\section{Conclusions}

In this paper, we propose a new variational model to restore blurred images corrupted by Poisson noise. Based on the good feature of high-order functional, we propose a model by adding an extra high-order functional term in the total variation model. Our model combines advantages of the firstorder and second-order total variation. It can substantially reduce the staircase effect, while preserving edges in the restored images. The issues of existence and uniqueness of a minimizer for this variational model is discussed. At last, we employ a gradient descent method to solve the associated Euler-Lagrange equation. The numerical experiments show that the proposed method outperforms some existing restoration methods in terms of the SNR, relative error, and SSIM map for Poisson noise removal problem. The comparisons between the reconstructed images obtained by the four methods show that the proposed one can alleviate the staircase effect significantly while preserve edges.

\section{Conflict of Interests}

All of the coauthors do not have a direct financial relation with the trademarks mentioned in our paper that might lead to a conflict of interests for any of the coauthors.

\section{Acknowledgment}

L. Jiang and J. Huang are supported by NSFC (10871034), X.-G. Lv and J. Liu are supported by NSFC (60973015 and 61170311), Sichuan Province Sci. \& Tech. Research 


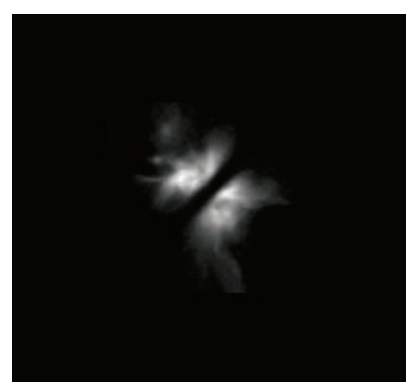

(a) True image

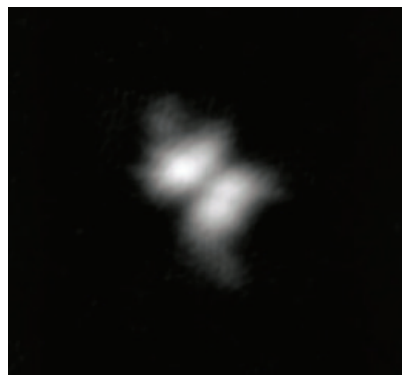

(e) Tikhonov

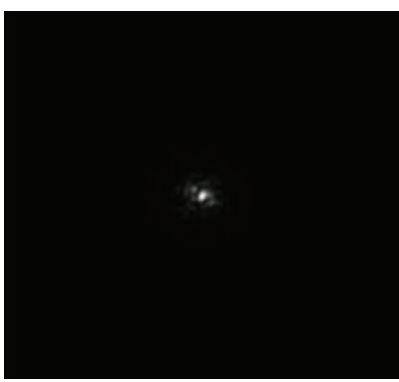

(b) PSF

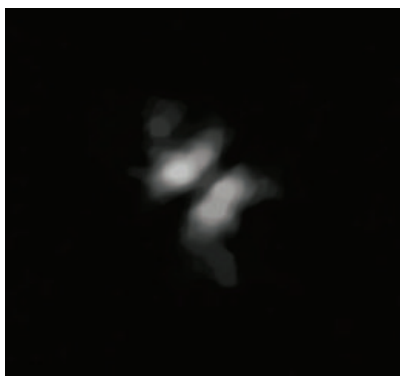

(f) TV

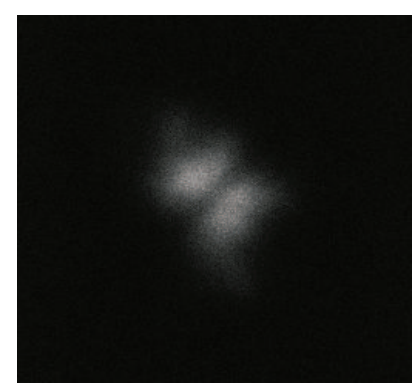

(c) Degraded image

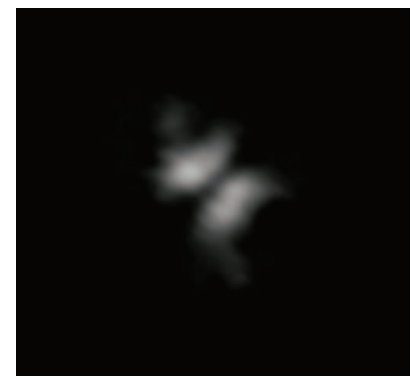

(g) HTV

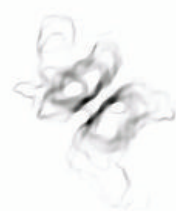

(d) Theta

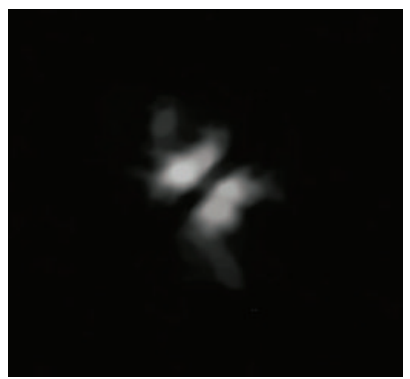

(h) Hybrid TV

Figure 5: Results of different methods for Example 3.

Project (2011JY0002 and 12ZC1802), and Chinese Universities Specialized Research Fund for the Doctoral Program (20110185110020).

\section{References}

[1] H. Andrew and B. Hunt, Digital Image Restoration,, Prentice Hall, Englewood Cliffs, NJ, USA, 1977.

[2] M. Bertero and P. Boccacci, Introduction to Inverse Problems in Imaging, Institute of Physics Publishing, London, UK, 1998.

[3] T. F. Chan and J. Shen, Image Processing and Analysis: Variational, PDE, Wavelet, and Stochastic Methods, SIAM, Philadelphia, Pa, USA, 2005.

[4] M. Banham and A. Katsaggelos, "Digital image restoration," IEEE Signal Processing Magazine, vol. 14, no. 2, pp. 24-41, 1997.

[5] L. Rudin, S. Osher, and E. Fatemi, "Nonlinear total variation based noise removal algorithms," Physical D, vol. 60, no. 1-4, pp. 259-268, 1992.

[6] A. Chambolle and P. L. Lions, "Image recovery via total variation minimization and related problems," Numerische Mathematik, vol. 76, no. 2, pp. 167-188, 1997.

[7] L. Rudin, P. L. Lions, and S. Osher, "Multiplicative denoising and deblurring: theory and algorithms," in Geometric Level Set Methods in Imaging, Vision, and Graphics, S. Osher and N. Paragios, Eds., pp. 103-119, Springer, New York, NY, USA, 2003.

[8] G. Aubert and J. F. Aujol, "A variational approach to removing multiplicative noise," SIAM Journal on Applied Mathematics, vol. 68, no. 4, pp. 925-946, 2008.

[9] Y. M. Huang, M. K. Ng, and Y. W. Wen, "A new total variation method for multiplicative noise removal," SIAM Journal on Imaging Sciences, vol. 2, no. 1, pp. 20-40, 2009.

[10] B. Chen, J. L. Cai, W. S. Chen, and Y. Li, "A multiplicative noise removal approach based on partial differential equation model," Mathematical Problems in Engineering, vol. 2012, Article ID 242043, 14 pages, 2012.

[11] S. Correia, M. Carbillet, P. Boccacci, M. Bertero, and L. Fini, "Restoration of interferometric images-I. The software package AIRY," Astronomy and Astrophysics, vol. 387, no. 2, pp. 733-743, 2002.

[12] A. Chambolle, "An algorithm for total variation minimization and applications," Journal of Mathematical Imaging and Vision, vol. 20, no. 1-2, pp. 89-97, 2004.

[13] T. Chan, S. Esedoglu, and F. Park, "Image decomposition combining staircase reduction and texture extraction," Journal of Visual Communication and Image Representation, vol. 18, no. 6, pp. 464-486, 2007.

[14] S. Becker, J. Bobin, and E. J. Candès, "NESTA: a fast and accurate first-order method for sparse recovery," SIAM Journal on Imaging Sciences, vol. 4, no. 1, pp. 1-39, 2011.

[15] A. Beck and M. Teboulle, "Fast gradient-based algorithms for constrained total variation image denoising and deblurring problems," IEEE Transactions on Image Processing, vol. 18, no. 11, pp. 2419-2434, 2009.

[16] Y. L. Wang, J. F. Yang, W. T. Yin, and Y. Zhang, "A new alternating minimization algorithm for total variation image reconstruction," SIAM Journal on Imaging Sciences, vol. 1, no. 3, pp. 248-272, 2008.

[17] J. M. Bioucas-Dias and M. A. T. Figueiredo, "Multiplicative noise removal using variable splitting and constrained optimization," IEEE Transactions on Image Processing, vol. 19, no. 7, pp. 1720-1730, 2010.

[18] S. Yun and H. Woo, "A new multiplicative denoising variational model based on $m$ th root transformation," IEEE Transactions on Image Processing, vol. 21, no. 5, pp. 2523-2533, 2012.

[19] H. Woo and S. Yun, "Alternating minimization algorithm for speckle reduction with a shifting technique," IEEE Transactions on Image Processing, vol. 21, no. 4, pp. 1701-1714, 2012. 
[20] M. Bertero, P. Boccacci, G. Desiderà, and G. Vicidomini, "Image deblurring with Poisson data: from cells to galaxies," Inverse Problems, vol. 25, no. 12, Article ID 123006, 26 pages, 2009.

[21] J. M. Bardsley and A. Luttman, "Total variation-penalized Poisson likelihood estimation for ill-posed problems," Advances in Computational Mathematics, vol. 31, no. 1-3, pp. 35-59, 2009.

[22] M. A. T. Figueiredo and J. M. Bioucas-Dias, "Restoration of Poissonian images using alternating direction optimization," IEEE Transactions on Image Processing, vol. 19, no. 12, pp. 31333145, 2010.

[23] S. Setzer, G. Steidl, and T. Teuber, "Deblurring Poissonian images by split Bregman techniques," Journal of Visual Communication and Image Representation, vol. 21, no. 3, pp. 193-199, 2010.

[24] D. Q. Chen and L. Z. Cheng, "Deconvolving Poissonian images by a novel hybrid variational model," Journal of Visual Communication and Image Representation, vol. 22, no. 7, pp. 643-652, 2011.

[25] I. Csiszár, "Why least squares and maximum entropy? An axiomatic approach to inference for linear inverse problems," The Annals of Statistics, vol. 19, no. 4, pp. 2032-2066, 1991.

[26] J. M. Bardsley and J. Goldes, “Techniques for regularization parameter and hyper-parameter selection in PET and SPECT imaging," Inverse Problems in Science and Engineering, vol. 19, no. 2, pp. 267-280, 2011.

[27] J. M. Bardsley and J. Goldes, "Regularization parameter selection methods for ill-posed Poisson maximum likelihood estimation," Inverse Problems, vol. 25, no. 9, Article ID 095005, 18 pages, 2009.

[28] D. Q. Chen and L. Z. Cheng, "Spatially adapted regularization parameter selection based on the local discrepancy function for Poissonian image deblurring," Inverse Problems, vol. 28, no. 1, Article ID 015004, 24 pages, 2012.

[29] G. Landi and E. L. Piccolomini, "NPTool: a Matlab software for nonnegative image restoration with Newton projection methods," Numerical Algorithms, 2012.

[30] A. Tikhonov and V. Arsenin, Solution of Ill-Poised Problems, Winston, Washington, DC, USA, 1977.

[31] G. Landi and E. L. Piccolomini, "An improved Newton projection method for nonnegative deblurring of Poisson-corrupted images with Tikhonov regularization," Numerical Algorithms, vol. 60, no. 1, pp. 169-188, 2012.

[32] J. M. Bardsley and C. R. Vogel, "A nonnegatively constrained convex programming method for image reconstruction," SIAM Journal on Scientific Computing, vol. 25, no. 4, pp. 1326-1343, 2003/04.

[33] J. M. Bardsley and N. Laobeul, “Tikhonov regularized Poisson likelihood estimation: theoretical justification and a computational method," Inverse Problems in Science and Engineering, vol. 16, no. 2, pp. 199-215, 2008.

[34] W. H. Richardson, "Bayesan-based iterative methods of image restoration," Journal of the Optical Society of America, vol. 62, no. 1, pp. 55-59, 1972.

[35] L. B. Lucy, "An iterative technique for the rectification of observed images," The Astronomical Journal, vol. 79, pp. 745754, 1974.

[36] V. Agarwal, A. V. Gribok, and M. A. Abidi, "Image restoration using $L_{1}$ norm penalty function," Inverse Problems in Science and Engineering, vol. 15, no. 8, pp. 785-809, 2007.

[37] G. Landi and E. L. Piccolomini, "An efficient method for nonnegatively constrained total variation-based denoising of medical images corrupted by Poisson noise," Computerized Medical Imaging and Graphics, vol. 36, no. 1, pp. 38-46, 2012.

[38] S. Lefkimmiatis, A. Bourquard, and M. Unser, "Hessian-based norm regularization for image restoration with biomedical applications," IEEE Transactions on Image Processing, vol. 21, no. 3, pp. 983-995, 2012.

[39] F. Li, C. M. Shen, J. S. Fan, and C. L. Shen, "Image restoration combining a total variational filter and a fourth-order filter," Journal of Visual Communication and Image Representation, vol. 18, no. 4, pp. 322-330, 2007.

[40] M. Lysker and X. C. Tai, "Iterative image restoration combining total varition minimization and a second-order functional," International Journal of Computer Vision, vol. 66, no. 1, pp. 518, 2006.

[41] K. Papafitsoros and C. B. Schönlieb, "A combined first and second order variational approach for image reconstruction," http://arxiv.org/abs/1202.6341.

[42] T. Chan, A. Marquina, and P. Mulet, "High-order total variation-based image restoration," SIAM Journal on Scientific Computing, vol. 22, no. 2, pp. 503-516, 2000.

[43] M. Lysaker, A. Lundervold, and X. C. Tai, "Noise removal using fourth-order partial differential equation with applications to medical magnetic resonance images in space and time," IEEE Transactions on Image Processing, vol. 12, no. 12, pp. 1579-1590, 2003.

[44] G. Steidl, "A note on the dual treatment of higher-order regularization functionals," Computing, vol. 76, no. 1-2, pp. 135148, 2006.

[45] H. Z. Chen, J. P. Song, and X. C. Tai, "A dual algorithm for minimization of the LLT model," Advances in Computational Mathematics, vol. 31, no. 1-3, pp. 115-130, 2009.

[46] T. Le, R. Chartrand, and T. J. Asaki, "A variational approach to reconstructing images corrupted by Poisson noise," Journal of Mathematical Imaging and Vision, vol. 27, no. 3, pp. 257-263, 2007.

[47] W. F. Zhou and Q. G. Li, "Poisson noise removal scheme based on fourth-order PDE by alternating minimization algorithm," Abstract and Applied Analysis, vol. 2012, Article ID 965281, 14 pages, 2012.

[48] Z. Wang, A. C. Bovik, H. R. Sheikh, and E. P. Simoncelli, "Image quality assessment: from error visibility to structural similarity," IEEE Transactions on Image Processing, vol. 13, no. 4, pp. 600612, 2004.

[49] J. G. Nagy, K. Palmer, and L. Perrone, "Iterative methods for image deblurring: a Matlab object-oriented approach," Numerical Algorithms, vol. 36, no. 1, pp. 73-93, 2004.

[50] P. C. Hansen, J. G. Nagy, and D. P. O’Leary, Deblurring Images: Matrices, Spectra, and Filtering, vol. 3 of Fundamentals of Algorithms, SIAM, Philadelphia, Pa, USA, 2006. 


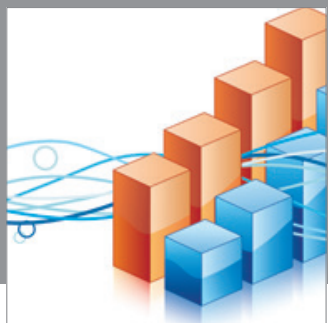

Advances in

Operations Research

mansans

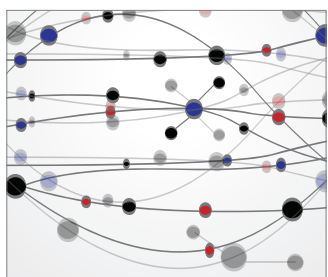

The Scientific World Journal
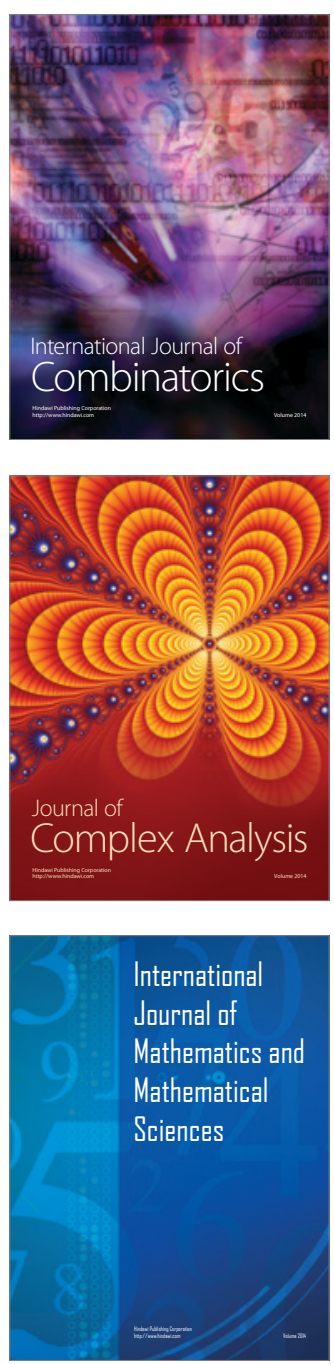
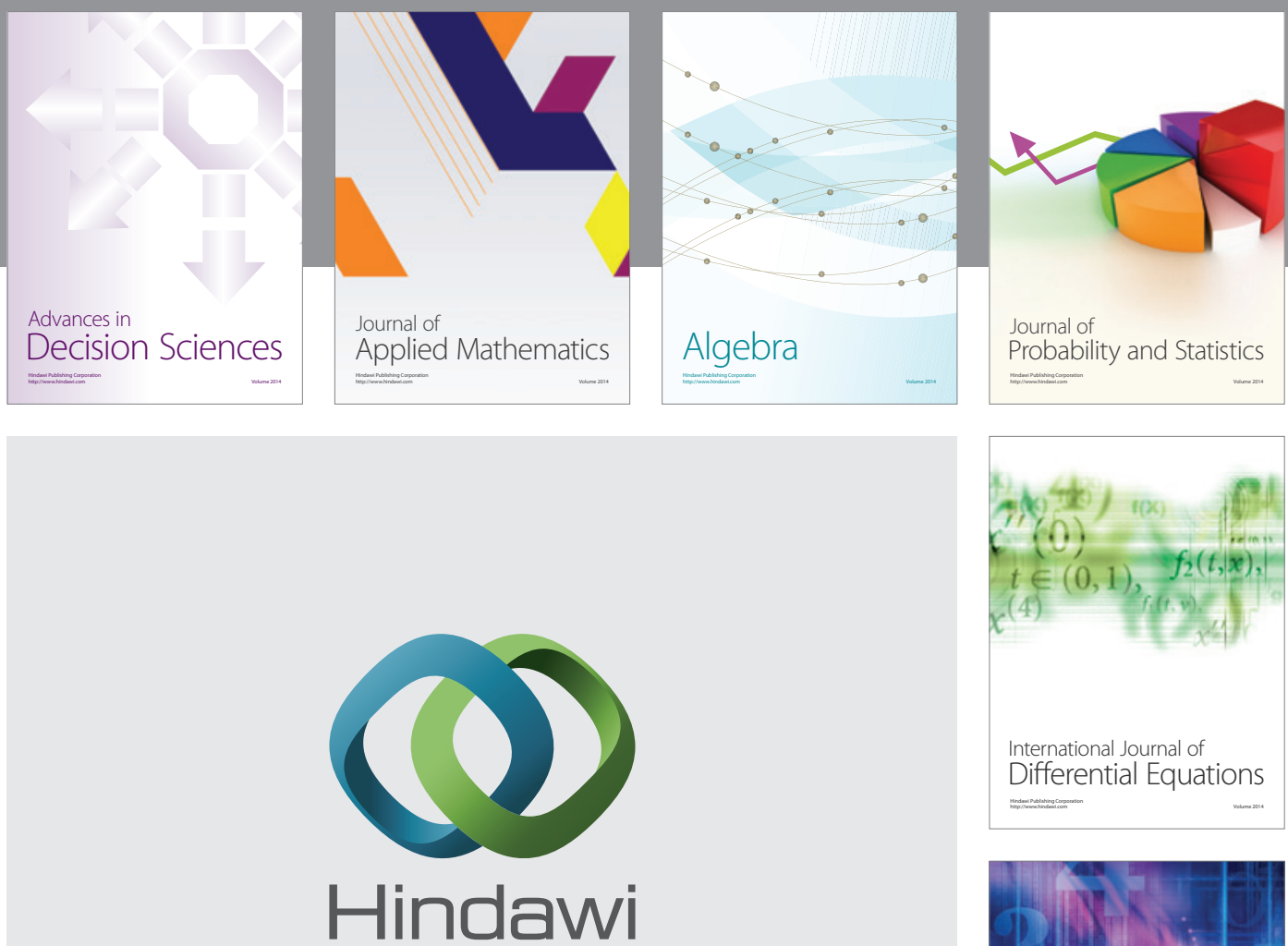

Submit your manuscripts at http://www.hindawi.com
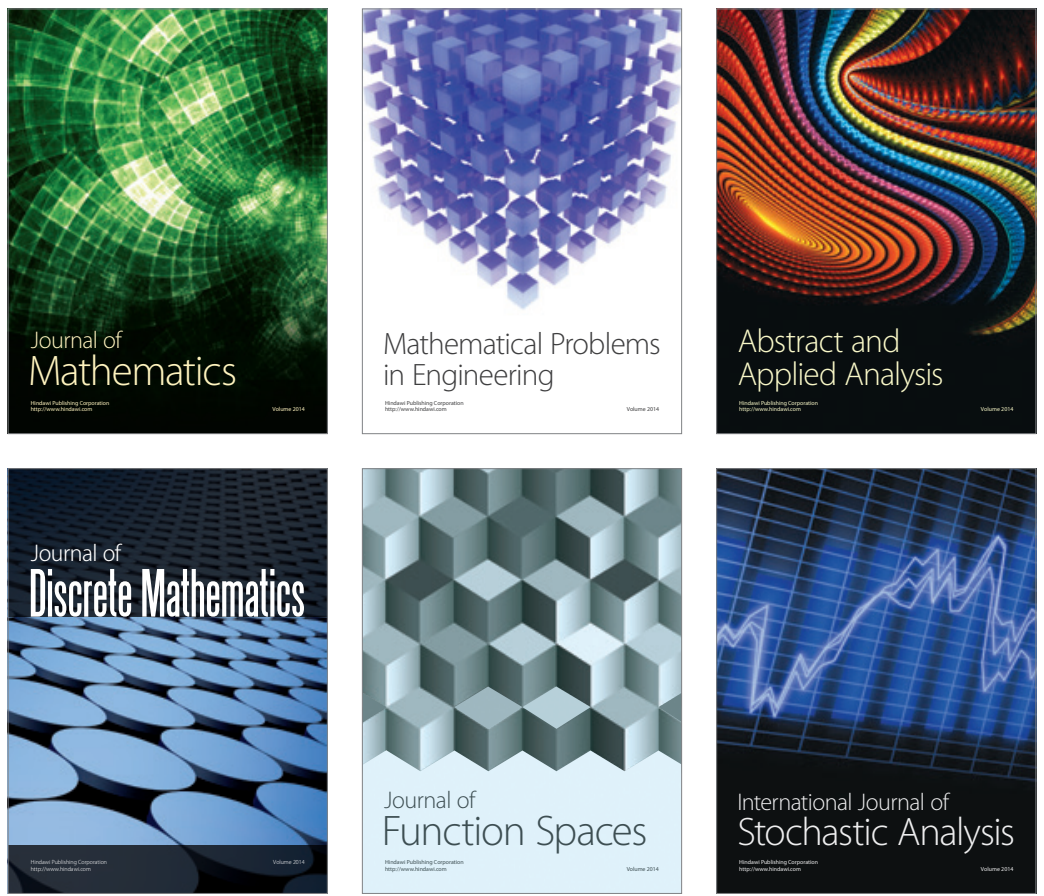

Journal of

Function Spaces

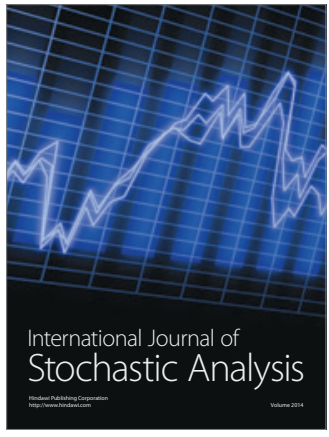

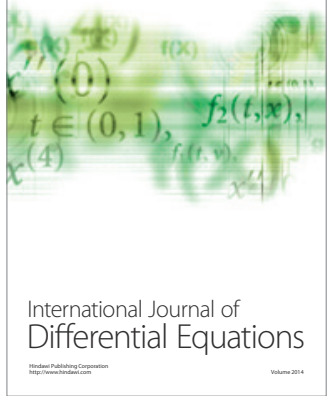
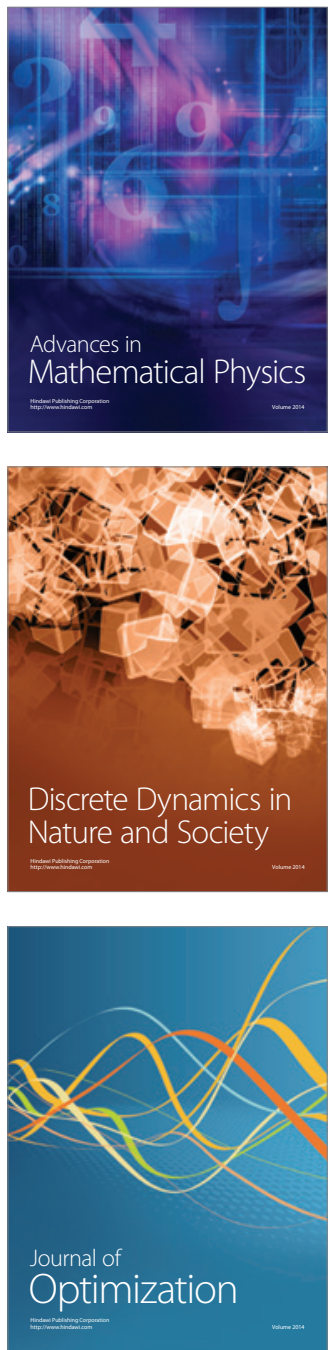